\title{
Transdiagnostic stepped care in mental health
}

\section{Shane PM Cross a,b and lan Hickie ${ }^{a}$}

a Brain and Mind Centre, University of Sydney, NSW, Australia.

bCorresponding author: shane.cross@sydney.edu.au

\section{Article history}

Publication date: April 2017

Citation: Cross SPM, Hickie I.

Transdiagnostic stepped care in mental health. Public Health Res Pract. 2017;27(2):e2721712. doi: http://dx.doi. org/10.17061/phrp2721712

\section{Key points}

- Current stepped-care models do not cater well for comorbidity or complexity in realworld clinical practice

- New transdiagnostic models are required to ensure that an adequate intensity of care is provided to those in need at the time they need it

- Technologically integrated models will free up service resources, and provide opportunities for comprehensive standardised assessment, shared treatment planning and outcomes monitoring

\section{Abstract}

A component of Australia's recent national reform agenda for mental health services is the directive to the Primary Health Networks to develop and implement stepped-care models of service delivery. The current guidance proposes that interventions are aligned to mild, moderate and severe illness categories. Other models in operation are tied to single disorders, such as depression. Both approaches have a number of limitations when applied to real-world, complex clinical practice, especially in primary care.

This article outlines some limitations of these models and argues for the development of a transdiagnostic model, based on developments in our work in primary care youth services, which can be generalised to adult populations. Such models aim to ensure that consumers receive the right intensity of care at the right time. The adjunct use of technology within services could also improve service accessibility and outcomes monitoring, and help to improve the efficiency of resource allocation based on consumer need.

\section{Introduction}

The Australian Government's mental health reform agenda is guided by the recommendations of the National Mental Health Commission National Review of Mental Health Programmes and Services. ${ }^{1}$ The government recently called on each of Australia's 31 Primary Health Networks (PHNs) to develop and implement a 'stepped-care approach' to mental health service delivery. The recommendation to PHNs is to stratify help seekers into different 'needs groups' along the continuum of at-risk, mild, moderate and severe illness categories, and to match intervention intensity with these needs. ${ }^{2}$ In primary care, general practitioners, in particular, will need to assign individuals to these broad categories without clear criteria of what exactly constitutes, for instance, a moderate versus a severe illness. This will result in wide variability of categorisation, both within and between PHNs, and ultimately disparate interventions recommended for those with the same sets of issues. Alternatively, other stepped-care models that focus on people with common single disorders, such as anxiety or depression, risk excluding people who have more complex needs. Our growing scientific understanding of the complex nature of mental ill health suggests that models that are either nonspecific or narrow are likely to result in the perpetuation of inadequate care provision for many consumers. 


\section{Stepped care in mental health}

The common objective of various stepped-care models is to rationalise the use of scarce resources, such as expensive psychological therapies delivered by trained and experienced clinicians for common disorders, typically anxiety and depression. ${ }^{3}$ Stepped-care models aim to offer low-intensity treatments as an early 'step', and then more intensive interventions if a person fails to benefit from the interventions listed as part of that step, purportedly to improve service access and efficiency. ${ }^{4,5}$ Under these models, low-intensity treatments involve minimal financial and time costs to the consumer ${ }^{4}$, clinicians and services.

The overall efficiency objectives are worthy and should be maintained; however, some other aspects of these models pose challenges:

1. Waiting list therapy. Because a number of consumers improve over time without treatment, many models advocate 'watchful waiting' as a first step. This results in treatment delays for many people who are experiencing significant distress or functional impairment. ${ }^{6}$ It is well established that most people living with a mental disorder have already delayed access to professional support, in some cases for many years. ${ }^{7}$ It would be difficult to imagine a cancer service directing consumers with established illness to a watchful waiting step.

2. The problem of comorbidity. Individuals rarely present to services with neatly packaged single disorders, on which most stepped-care models are based. It has been well established that comorbidity is the rule rather than the exception. ${ }^{8}$ Forcing individuals with comorbidities into stepped-care approaches that focus on single disorders ignores the complexity of their needs, arguably resulting in undertreatment.

3. Whether only those with full-threshold disorders have a need for intervention. The boundary between 'normality' and disorder is poorly defined ${ }^{9}$, and many have argued that these boundaries currently exclude large numbers of people who are functionally impaired or distressed from accessing care because they narrowly miss out on meeting arbitrary full-threshold diagnostic criteria. The distress and functional impairment caused by subthreshold disorders (which meet most, but not all, features to qualify for a diagnosis under the Diagnostic and statistical manual of mental disorders or the International Statistical Classification of Diseases and Related Health Problems) are roughly equivalent to those associated with full-threshold disorders ${ }^{10}$ and therefore require intervention.

4. The nonstatic nature of mental disorders. Severe mental disorders do not develop spontaneously. The more common experience is a long history of impairing and distressing symptoms many years before the establishment of a 'diagnosable' severe disorder. Many disorders are sequentially comorbid, and recurrent or chronic, and exist on a continuum. ${ }^{11}$ Disorders tend to develop over a period of time when complex interactions occur between individual risk factors, across and between biological, psychological and social domains. ${ }^{12}$ There is also accumulating evidence that mental disorders can progress to more severe states over time, whether as a worsening of the current syndrome or as a progression to a different and more severe illness class. ${ }^{13}$ The earliest observable expressions of severe psychopathology are often a mix of nonspecific anxiety and depressive symptoms, which gradually develop into more distinct syndromes. ${ }^{12}$

\section{Stage-based stepped care}

New models of mental health service delivery need to be responsive to these complexities. Such models need to cope with comorbidity, impairment resulting from subthreshold disorders and the potential trajectory of disorders, and, most importantly, be able to match intervention intensity to the severity of concerns. Clinical staging is a framework that copes with these issues and can be used alongside the traditional diagnostic system to guide better treatment decisions..$^{12,14,15}$ Staging models define where an individual sits on a continuum, from an at-risk but asymptomatic state (stage 0) through to a persistent, chronic and unremitting disorder state (stage 4). ${ }^{15}$ This framework is readily applied to people who present for healthcare, and clearly differentiates those who are typically younger and in early phases (stages 1a 'help seeking' or 1b 'attenuated syndromes') from those who are typically older and have reached a higher threshold for disorder (stage 2 and above; Figure 1). The model has support from recent studies in young people demonstrating its clinical and service-level utility. ${ }^{14,16,17}$

\section{Examples from youth services incorporating clinical staging}

The vast majority of mental disorders have their onset by adolescence and early adulthood. ${ }^{18}$ Disorder-based stepped-care models have limited usefulness in these populations, primarily because of the high rates of subthreshold disorders in young people. ${ }^{10}$ Our preliminary longitudinal work using this clinical staging system in primary care settings has indicated that approximately $20 \%$ of those rated as stage $1 \mathrm{~b}$ at initial assessment progress to a later stage, and typically do so within 12 months. ${ }^{14}$ When examining the service engagement, treatments provided and clinical outcomes, we found that these were strongly associated with clinical stage, such that those at stage $1 \mathrm{~b}$ made more clinical appointments, received more intensive intervention, and had poorer clinical outcomes over the same period than those at stage 1a. ${ }^{16,17}$ We have since developed a transdiagnostic 
stage-based model of care for use in primary-level youth mental health services ${ }^{19}$, which can be readily applied to adults.

\section{Key features of a transdiagnostic staging model}

The proposed key features of a transdiagnostic stagebased stepped-care approach are outlined below.

\section{Assessment and staging}

Thorough initial broad and holistic screening is followed by a more targeted mental health assessment for those who respond positively to key screening questions. We previously outlined the key features of the assessment process under a staging model specific to centre-based services. ${ }^{19}$ The primary purpose of assessment is to match level of need with treatment intensity across six functional domains: mental health and clinical stage; education, training and employment status; risk of injury, self-harm and suicide; use of alcohol and other drugs; quality of physical health; and quality of family and social relationships. Additionally, assessing a person's strengths and personal or external resources aids in personalised treatment planning. The allocation of a clinical stage is based on published criteria ${ }^{14}$, ideally in consultation with professional peers or supported by technology.

\section{Treatment intensity}

The intensity of the intervention should match the level of need at the outset as determined by stage, considering the risk-to-benefit ratio of the proposed intervention. Early-stage treatments carry less risk, and are less time intensive for the consumer and the clinician than laterstage treatments (Figure 1). The spectrum of services matched against need (from lowest to highest intensity) comprises online psychoeducation, self-managed online interventions, clinician-supported online interventions, group-based psychological interventions, brief face-toface psychological therapy, longer-term psychological therapy, case management, and first- and secondline medication (Figure 1). Early-stage psychological interventions should be transdiagnostic. ${ }^{20}$ Intensity can be defined in terms of financial cost (both service level and consumer), consumer requirements to physically attend, the required frequency of attendance, the number of professionals involved, the length of the episode of care and the prescription of psychotropic medication.

\section{Intervening in other risk factors}

Parallel intervention should be provided for co-existing risk factors that are linked to poor outcome and worsening mental health, such as unemployment, alcohol and other drug use, poor physical health and social disconnection. At times, it is appropriate for these domains to be the primary focus of intervention.

\section{Assertive monitoring of outcomes}

Proactively monitoring treatment outcomes is critical, and consumers would ideally have direct and transparent access to their own data to allow shared treatment planning with clinicians. Treatment progress and outcomes in care should be measured routinely and systematically, ideally supported by efficient digital technologies. A brief reassessment of the stage, other functional domain changes and psychometrics helps determine the intervention step: whether a person no longer requires intense treatment, requires an increase in treatment intensity within a stage, or requires a step up in treatment intensity because they have transitioned to a higher stage.

Figure 1 Recommended interventions by clinical stage

Increasing severity, complexity or chronicity of condition

Increasing intensity, cost and time in care. Decreasing number of people treated.

\begin{tabular}{|c|c|c|c|}
\hline $\begin{array}{c}\text { Stage } \\
\mathbf{1 a}\end{array}$ & Stage & Stage & $\begin{array}{c}\text { Stage } \\
\mathbf{3}\end{array}$ \\
\hline
\end{tabular}


Providing the required services at scale: technologically integrated service organisation

Most primary care services use technology such as electronic medical records, booking systems, SMS reminders and information exchange between providers. Technology will and should continue to evolve to better meet the needs of consumers, while at the same time improving service efficiency and effectiveness. Online standardised and comprehensive assessment of consumer need could overcome the barriers of opening hours and location of services. With more efficient assessment processes, clinicians can spend more time delivering interventions. ${ }^{6}$ The information obtained from these comprehensive assessments can then be used by consumers in partnership with their clinicians to guide a range of interventions matched to their needs. The effectiveness of these interventions would be monitored by these technologies in real time, and such routine outcome monitoring would assist consumers and clinicians to determine whether intervention intensity should step up or down. These data would also be of use to service providers when reporting on servicelevel outcomes.

Reform of the mental health system to provide interventions based on severity and need across the life span will require clinical models that are specific, flexible and inclusive. Substantial service restructuring, improving the technology already used in services, and providing consumers with their own health information to track progress will fundamentally reform the way in which services are delivered to better meet need.

\section{Competing interests}

IH receives money from the National Mental Health Commission for his role as a Mental Health Commissioner, and from Medibank Private as a member of its Medical Advisory Panel. He has previously received payment as a member of the Bupa Australia Medical Advisory Board, and for lectures from Servier, Janssen, AstraZeneca and Pfizer.

\section{Author contributions}

$\mathrm{SC}$ and $\mathrm{IH}$ both conceived of and contributed to the paper.

\section{References}

1. National Mental Health Commission. Contributing lives, thriving communities: report of the national review of mental health programmes and services. Canberra: NMHC; 2014 [cited 2017 Apr 7]. Available from: www. mentalhealthcommission.gov.au/our-reports/contributinglives,-thriving-communities-review-of-mental-healthprogrammes-and-services.aspx

2. Australian Government Department of Health. PHN primary mental health care flexible funding pool implementation guidance: stepped care. Canberra: Commonwealth of Australia; 2016 [cited 2017 Mar 7]. Available from: www.health.gov.au/internet/main/ publishing.nsf/content/2126B045A8DA90FDCA257F6500 018260/\$File/1PHN\%20Guidance\%20-\%20Stepped\%20 Care.PDF

3. National Collaborating Centre for Mental Health (UK). Depression: the treatment and management of depression in adults (updated edition). Leicester, UK: British Psychological Society; 2010.

4. van Straten A, Hill J, Richards D, Cuijpers P. Stepped care treatment delivery for depression: a systematic review and meta-analysis. Psychol Med. 2015;45(2):231-46.

5. Bower P, Gilbody S. Stepped care in psychological therapies: access, effectiveness and efficiency. Narrative literature review. Br J Psychiatry. 2005;186(1):11-7.

6. Richards DA, Bower P, Pagel C, Weaver A, Utley M, Cape J, et al. Delivering stepped care: an analysis of implementation in routine practice. Implement Sci. 2012;7:3.

7. Kohn R, Saxena S, Levav I, Saraceno B. The treatment gap in mental health care. Bull World Health Organ. 2004;82(11):858-66.

8. First MB. Mutually exclusive versus co-occurring diagnostic categories: the challenge of diagnostic comorbidity. Psychopathology. 2005;38(4):206-10.

9. Kendell R, Jablensky A. Distinguishing between the validity and utility of psychiatric diagnoses. Am J Psychiatry. 2003;160(1):4-12.

10. Roberts RE, Fisher PW, Turner JB, Tang M. Estimating the burden of psychiatric disorders in adolescence: The impact of subthreshold disorders. Soc Psychiatry Psychiatr Epidemiol. 2015;50(3):397-406.

11. Caspi A, Houts RM, Belsky DW, Goldman-Mellor SJ, Harrington $\mathrm{H}$, Israel $\mathrm{S}$, et al. The $\mathrm{p}$ factor: one general psychopathology factor in the structure of psychiatric disorders? Clin Psychol Sci. 2014;2(2):119-37.

12. Hickie I, Scott J, Hermens D, Scott E, Naismith S, Guastella A, et al. Clinical classification in mental health at the cross-roads: which direction next? BMC Med. 2013;11:125. 
13. Copeland WE, Shanahan L, Costello EJ, Angold A. Childhood and adolescent psychiatric disorders as predictors of young adult disorders. Arch Gen Psychiatry. 2009;66(7):764-72.

14. Hickie IB, Scott EM, Hermens DF, Naismith SL, Guastella AJ, Kaur M, et al. Applying clinical staging to young people who present for mental health care. Early Interv Psychiatry. 2013;7(1):31-43.

15. McGorry PD, Purcell R, Hickie IB, Yung AR, Pantelis C, Jackson HJ. Clinical staging: a heuristic model for psychiatry and youth mental health. Med J Aust. 2007;187(7 Suppl):S40-2.

16. Cross SPM, Hermens DF, Scott J, Salvador-Carulla L, Hickie IB. Differential impact of current diagnosis and clinical stage on attendance at a youth mental health service. Early Interv Psychiatry. 2016.
17. Cross SPM, Hermens DF, Hickie IB. Treatment patterns and short-term outcomes in an early intervention youth mental health service. Early Interv Psychiatry. 2016;10(1):88-97.

18. Kessler RC, Berglund P, Demler O, Jin R, Merikangas KR, Walters EE. Lifetime prevalence and age-of-onset distributions of DSM-IV disorders in the National Comorbidity Survey Replication. Arch Gen Psychiatry. 2005;62(6):593-602.

19. Cross SP, Hermens DF, Scott EM, Ottavio A, McGorry PD, Hickie IB. A clinical staging model for early intervention youth mental health services. Psychiatr Serv. 2014;65(7):939-43.

20. Kazdin AE. Evidence-based psychotherapies II: changes in models of treatment and treatment delivery. S Afr J Psychol. 2015;45(1):3-21.

\section{Copyright: (c)()(2)}

